\title{
Viabilidade do uso de turbinas hidrocinéticas na Plataforma Continental Sul-Sudeste Brasileira.
}

\author{
Feasibility of the usage of hydrokinetic turbines in the South-Southeast Brazilian Continental \\ Shelf. \\ E. P. Kirinus ${ }^{1 *}$; W. C. Marques ${ }^{2}$

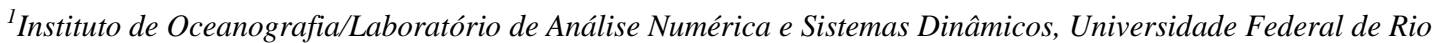 \\ Grande, 96203-900, Rio Grande-RS, Brasil. \\ ${ }^{2}$ Instituto de Matemática, Estatística e Física/Laboratório de Análise Numérica e Sistemas Dinâmicos, Universidade \\ Federal de Rio Grande, 96203-900, Rio Grande-RS, Brasil.
}

*ekirinus@gmail.com

(Recebido em 23 de setembro de 2014; aceito em 29 de dezembro de 2014)

\begin{abstract}
Devido à grande atenção dada ao desenvolvimento de novas fontes de captação energéticas, houve também, um aumento na diversidade de tecnologias existentes para a obtenção energética de forma limpa e sustentável. Na Plataforma Continental Sul-Sudeste do Brasil, foram realizadas simulações hidrodinâmicas com o sistema de modelagem numérica TELEMAC3D, juntamente com um módulo de conversão de energia, para a simulação de turbinas hidrocinéticas, que vem sendo desenvolvido na FURG. Dentre os locais estudados, a região de Santa Catarina apresenta as melhores características de intensidade de corrente e batimetria para a conversão de energia das correntes. Os resultados das simulações demonstraram que essa região apresenta valores integrados de potência que podem alcançar 4,71 MW durante o período analisado de 120 dias, com média de conversão de aproximadamente 45 $\mathrm{kW} / \mathrm{dia}$.
\end{abstract}

Palavras-chave: TELEMAC3D, Potência energética, Energias renováveis.

Due to the intensive attention granted to the investigation of new sources of energy conversion, there is also, an effort to promote the development of new technologies for the conversion of renewable and clean energy has being strengthened. In the South-Southeast Brazilian Continental Shelf, hydrodynamic simulations were performed using the TELEMAC3D model, coupled with the energy conversion module for the simulation of the hydrokinetic turbines under development at FURG. Within the studied points, the Santa Catarina region showed the best characteristics, such as bathymetry and current intensity, for the installment of turbines. The simulation results showed high capacity of generating power, with integrated power reaching $4.71 \mathrm{MW}$ with average conversion around $45 \mathrm{~kW} /$ day during the 120 days analyzed.

Keywords: TELEMAC3D, Power, Renewable energies.

\section{INTRODUÇÃO}

O rápido aumento global das necessidades energéticas mantém uma escalada da concorrência sobre os recursos disponíveis e também o aumento dos preços da energia repassada à população. Atualmente, a sociedade precisa enfrentar grandes desafios para fornecer energia para a comunidade por intermédio de soluções sustentáveis e preservando a qualidade ecológica.

De uma forma geral, os recursos não renováveis convencionalmente têm a tendência para a deterioração da qualidade ecológica, liberando uma quantidade excessiva de dióxido de carbono $\mathrm{CO} 2$ e outros poluentes na atmosfera. Por outro lado, fontes renováveis de energia como: a solar, a eólica, a biomassa, a térmica dos oceanos e a energia mecânica dos oceanos (proveniente das ondas e correntes), são alternativas emergentes, que podem suprir uma parte da crescente demanda de energia com menores impactos ao meio ambiente [1].

As regiões costeiras estão entre as regiões de crescimento mais rápido do mundo, gerando fortes pressões populacionais, como para o abastecimento de água e energia. Em escala global, o panorama não é muito diferente, pois mais da metade da população dos Estados Unidos, por exemplo, vive a $80 \mathrm{~km}$ do litoral, próxima aos recursos energéticos marinhos [2]. O crescimento da população nos estados ao longo da costa sudeste (Carolina do Norte, Carolina do Sul, 
Geórgia e Flórida) exige um maior fornecimento anual de energia enquanto a crescente pressão sobre o ambiente se acentua.

A energia das correntes oceânicas pode ser transformada em energia elétrica utilizando conversores com tecnologia semelhante à utilizada em conversores eólicos, através de um rotor submerso que é forçado a girar pelo fluido que passa ao seu redor. Segundo [3], estes equipamentos de captação de energia aplicados para corpos d'água podem ser encontrados no formato de sistemas convencionais com a utilização de turbinas e sistemas não convencionais sem a utilização de turbinas. Segundo estes autores, com algumas exceções, cada equipamento foi desenvolvido para aplicação em determinado local, correspondendo às características físicas e espaciais do ambiente a ser implementado.

A energia das correntes pode ser captada de duas formas: através da energia potencial (devido a variações no nível do mar) ou da energia cinética, a qual consiste em aproveitar essencialmente a velocidade das correntes e de massas de água associadas. A técnica utilizada, de uma forma geral, pode ser definida como eólica submarina, tendo aproximadamente os mesmos princípios de funcionamento e só diferindo o fluido com a qual interagem.

Gorlov [4], estudando a circulação gravitacional e algumas aplicações para a extração de energia, concluiu que estas são fontes substanciais de energia renovável para as futuras gerações. A agência Canadense de Hidrologia CHC (Canadian Hydraulics Institute) realizou um estudo utilizando modelagem numérica que mapeou a costa canadense produzindo um mapa dos recursos energéticos de fonte marinha da região, considerando as ondas geradas pelo vento e as correntes de maré [5]. Neste estudo, foi verificado que a potência elétrica média disponível nos 190 pontos analisados equivaleria a aproximadamente $63 \%$ da demanda de energia canadense.

Um estudo similar foi realizado por [6] nos Estados Unidos, onde os autores identificaram as regiões com os maiores potenciais para a utilização de hidroenergia, considerando como de baixo potencial as regiões com potência média anual de até $1 \mathrm{MW} / \mathrm{ano}$, e de médio e alto potencial, as regiões com potência média anual entre 1 e 30 MW/ano. De acordo com [3], a energia dos fluxos de rios e estuários, correntes de marés e outros canais de água artificiais podem ser considerados como uma fonte viável de energia renovável. Em [7] foi investigado o potencial energético das ondas geradas pelo vento e correntes de maré na costa sudeste dos Estados Unidos, através da análise de dados e modelagem numérica, onde verificou um possível potencial de conversão que varia entre 1,0 e 3,0 MW/ano.

Desenvolvimentos recentes demonstraram uma redução dos impactos ambientais quando o dispositivo de conversão é completamente submerso. Através de diversos estudos independentes, foi demonstrado que o impacto sobre os peixes e mamíferos marinhos é baixo devido ao lento movimento de tais dispositivos [8]. Existem muitas tecnologias emergindo para converter a energia destes recursos em energia elétrica. Entretanto, o primeiro passo, para a conversão destes recursos consta no mapeamento abrangente dos mesmos, onde é esperado determinar a viabilidade e a acessibilidade das fontes, assim como o nível de conversão que pode ser atingido.

Dentro desta premissa, o principal objetivo deste trabalho é estudar, através de um modelo de circulação oceânica, a viabilidade da utilização dos conversores de energia do tipo turbina na região sul e sudeste da Plataforma Continental Sul-Sudeste Brasileira.

\section{1. Área de estudo}

A área de estudo deste trabalho se estende do litoral sul do estado do Rio de Janeiro $\left(22^{\circ} \mathrm{S}\right)$ até o Chuí $\left(33^{\circ} \mathrm{S}\right)$ (Figura 1). Uma das principais feições oceanográficas observadas é a Corrente do Brasil, que após ser originada na linha do equador, se desloca para sul ao longo da plataforma continental brasileira carregando Água Tropical e a Água Central do Atlântico Sul.

O fluxo sentido sul da Corrente do Brasil encontra o fluxo norte da Corrente das Malvinas entre as latitudes de 33 e $38^{\circ} \mathrm{S}$ [9]. A região de encontro entre a Corrente do Brasil e a Corrente das Malvinas é conhecida como Confluência Brasil-Malvinas, ocorrendo em torno de $38^{\circ} \mathrm{S}$. A colisão de uma corrente sul carregando água tropical e uma corrente norte carregando água 
subantártica resulta em um padrão de circulação superficial dominado por um forte gradiente termohalino com a formação de meandros e vórtices [9-10].

Devido a estas instabilidades, a Confluência Brasil Malvinas (CBM) pode ser considerada com uma das regiões mais energéticas dos oceanos [10-12]. Segundo Oliveira [13], a interação entre o fluxo médio dos giros de larga-escala e atividade de meso-escala é importante para o balanço de energia, especialmente em áreas dos oceanos onde as correntes de contorno oeste se separam da costa.
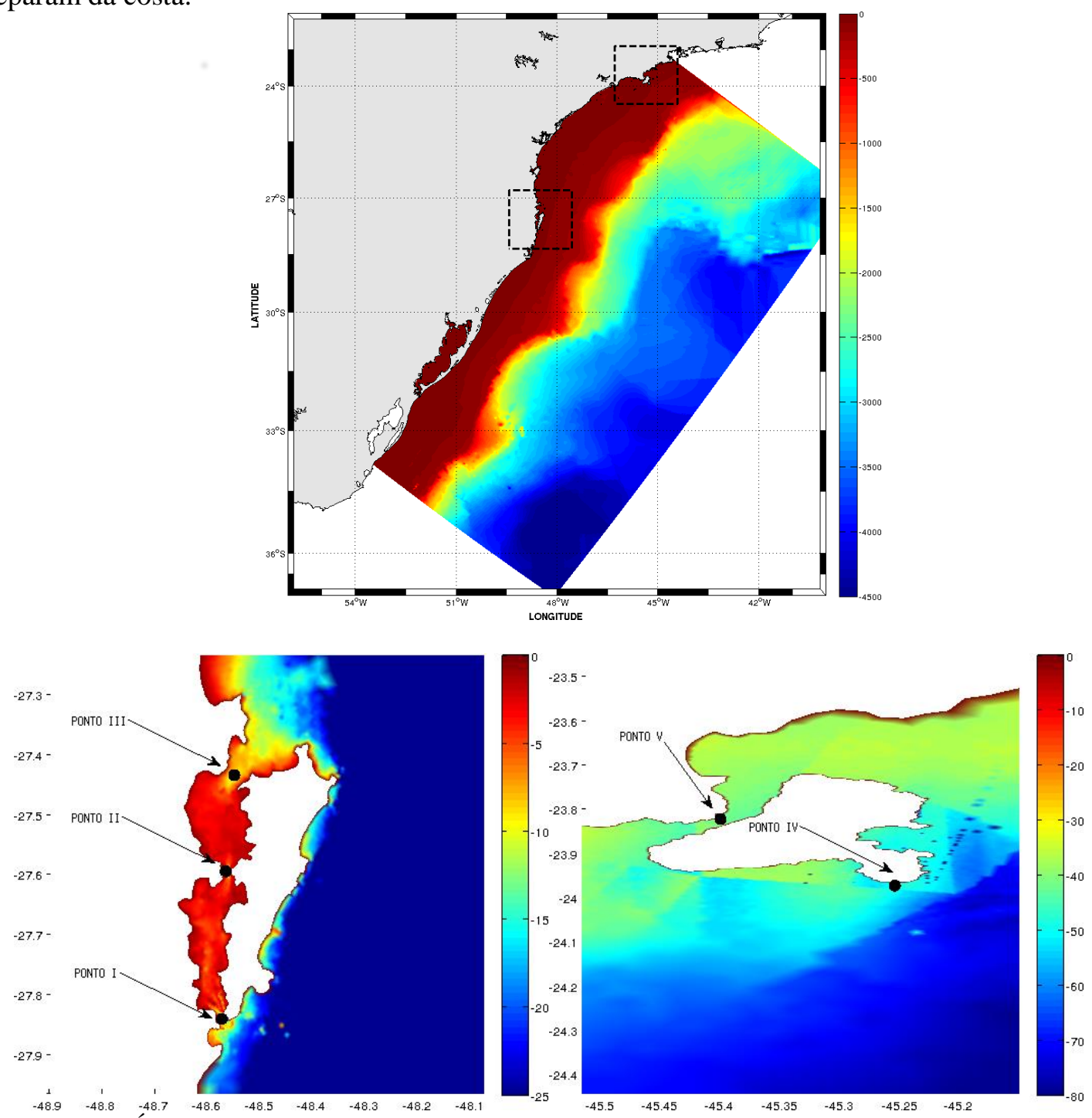

Figura 1: Área de estudo. Em destaque as duas regiões de interesse, a Ilha de Florianópolis em Santa Catarina, e a Ilhabela em São Paulo. A batimetria da área de estudo se estende até aproximadamente $4.500 \mathrm{~m}$. Os pontos demonstram os locais de interesse.

\section{MATERIAL E MÉTODOS}

\subsection{Modelo numérico hidrodinâmico}

O modelo numérico para simulações hidrodinâmicas utilizado foi o TELEMAC3D, (www.opentelemac.org). Esse modelo utiliza as equações de Navier-Strokes considerando as variações locais na superfície livre do mar, considerando a densidade constante, a pressão hidrostática e as aproximações de Boussinesq. O modelo é baseado em técnicas de elementos finitos para resolver a equação hidrodinâmica [19] e conta com o sistema de coordenadas sigma para discretização vertical. 
Um passo de tempo de 90s e um parâmetro de Coriolis variável de acordo com a latitude da malha numérica do modelo foram utilizados. Seguindo a metodologia de [15-16], um modelo de Smagorinsky foi utilizado para a modelagem da turbulência horizontal e um modelo de comprimento de mistura modificado para regiões costeiras foi aplicado a modelagem da turbulência vertical.

A discretização vertical consiste da utilização de 15 níveis sigma igualmente espaçados em um domínio numérico de 115.320 nós. É importante ressaltar que, a parametrização utilizada neste trabalho segue os estudos de [15-18] onde o sistema de modelagem foi calibrado e validado para a realização de estudos da hidrodinâmica e morfodinâmica na plataforma continental do Sul do Brasil.

\subsection{Módulo de conversão de energia}

O módulo de conversão de energia utiliza a equação padrão de turbinas para calcular a energia elétrica gerada, em Watts (W), a partir da velocidade do fluxo da corrente incidente nos conversores:

$$
P(\mathrm{~W})=1 / 2 \rho \mu A v^{3}
$$

onde: $P(\mathrm{~W})$ é a potência gerada em Watts, $\rho\left(\mathrm{kg} / \mathrm{m}^{3}\right)$ é a massa específica do fluido, $\mu$ (adimensional) é o coeficiente de eficiência da turbina, $A\left(\mathrm{~m}^{2}\right)$ é a área do conversor e $v(\mathrm{~m} / \mathrm{s})$ é a velocidade. A Tabela 1 indica os parâmetros utilizados pelo módulo de conversão de energia para os locais estudados.

Utilizando o princípio da conservação de energia, a velocidade da corrente é obtida em cada intervalo de tempo através do modelo hidrodinâmico que transfere para o módulo de conversão de energia. Uma parte da velocidade é convertida em energia elétrica, a velocidade é então atualizada para manter o balanço de energia do modelo hidrodinâmico, TELEMAC3D.

Tabela 1: Parâmetros técnicos das turbinas.

\begin{tabular}{ccc}
\cline { 2 - 3 } Parâmetro & SC & SP \\
\hline Tipo de turbina & Helicoidal [19] & Axial [20] \\
Velocidade de início de & $0,2 \mathrm{~m} / \mathrm{s}$ & $0,2 \mathrm{~m} / \mathrm{s}$ \\
$\begin{array}{c}\text { movimento } \\
\text { Velocidade nominal }\end{array}$ & $1,5 \mathrm{~m} / \mathrm{s}$ & $1,5 \mathrm{~m} / \mathrm{s}$ \\
Coeficiente de eficiência & 0,35 & 0,45 \\
da turbina $(\mu)$ & $170 \mathrm{~kW}$ & $352 \mathrm{~kW}$ \\
Potência nominal & $14 \mathrm{~m}$ & ---- \\
Altura da turbina & $10 \mathrm{~m}$ & $12 \mathrm{~m}$ \\
Raio da turbina & & \\
\hline
\end{tabular}

Como a região de interesse no estado de Santa Catarina apresenta profundidade de $15 \mathrm{~m}$ ao sul e norte, e mais de $30 \mathrm{~m}$ de profundidade no centro, foi idealizado uma turbina helicoidal de $14 \mathrm{~m}$ de altura e $10 \mathrm{~m}$ de raio. Por outro lado, a região de Ilhabela apresenta, nos dois pontos selecionados uma batimetria superior a $40 \mathrm{~m}$, permitindo a utilização de turbinas axiais.

Para o estudo do potencial de conversão foi realizada uma simulação de 120 dias e o período simulado compreende entre 01 de janeiro e 30 de abril de 2004.

\subsection{Condições Iniciais e de Contorno}

O contorno oceânico é prescrito por: marés astronômicas, elevação do nível da água do mar, salinidade e temperatura. Os valores de salinidade e temperatura utilizados nas condições iniciais foram obtidos do modelo global HYbrid Coordinate Ocean Model (HYCOM http://hycom.org/).

A maré astronômica é calculada através da assimilação de dados do modelo global TOPEX/Poseidon Global Inverse Solution (http://volkov.oce.orst.edu/tides/global.html), 
modelo global de marés oceânicas que contempla as 29 principais constituintes de maré para definir a condição de contorno de correntes superficiais.

No contorno superficial, as variações espaço-temporais do vento foram representadas através de dados da página do National Oceanic \& Atmospheric Administration (NOAA), interpolados e aplicados a cada nó do domínio do modelo numérico. De forma complementar, os dados de temperatura do ar junto à superfície do oceano foram obtidos da página da NOAA, e forçados de forma similar aos ventos, possibilitando que os processos de troca de calor com a atmosfera fossem levados em consideração no modelo.

\section{RESULTADOS E DISCUSSÃO}

Os possíveis locais para instalação de turbinas na Plataforma Continental Sul-Sudeste Brasileira foram avaliados através da modelagem numérica tridimensional. A potência gerada pelos conversores de corrente foi estimada pelo módulo de conversão de energia, e os resultados foram analisados de acordo com os locais de maior produção energética da região costeira. Nestas regiões foram obtidas séries temporais de potência elétrica convertida, as quais foram analisadas de forma a obter as escalas de variabilidade energética dominantes para a região.

Para identificar zonas potenciais para a instalação de turbinas hidrocinéticas, é necessária a confluência de fatores como batimetria adequada, velocidade alta de fluxo incidente e por fim, a correta definição da direção do fluxo incidente à região de interesse. Sendo assim, a escolha da turbina adequada para cada região se alterna devido aos fatores acima citados. No litoral de Santa Catarina, a turbina de hélice helicoidal [19] aparece como opção mais adequada, pois esta turbina pode ser instalada em regiões rasas (15-25 m de profundidade) sendo capaz de gerar energia a partir de rotações unidirecionais independentes da direção da corrente que atinge o rotor [21].

Por outro lado, a região de Ilhabela-SP, apresenta uma profundidade elevada (de 30 a $50 \mathrm{~m}$ ), propiciando a instalação de turbinas axiais unidirecionais, que são bastante utilizadas para aplicações em fluxos controlados ou constantes como correntes de marés e rios canalizados, além de possuir maior coeficiente de eficiência. Os parâmetros utilizados para definir as turbinas em cada região estão presentes na Tabela 1.

\subsection{Litoral de Santa Catarina}

Para identificar as maiores intensidades de corrente, foram criados mapas de velocidade média da corrente e seu desvio padrão durante os 120 dias de simulação. A Figura 2a apresenta a velocidade média de corrente variando de zero a $0,45 \mathrm{~m} / \mathrm{s}$, enquanto a Figura $2 \mathrm{~b}$ apresenta $\mathrm{o}$ desvio padrão da velocidade de corrente, que varia de zero a $0,40 \mathrm{~m} / \mathrm{s}$. Na Figura 2 é possível identificar três locais de interesse devido à intensidade da velocidade média. As regiões em cor preta na Figura 2 demonstram baixas ou nenhuma intensidade do parâmetro estudado.

A potência energética estimada para as turbinas helicoidais para essas regiões está apresentada na Figura 3 como potência convertida através da média, desvio padrão e máximo em $\mathrm{kW} / \mathrm{dia}$, além da potência integrada em MW/120 dias.

Podemos observar que a região central (próximo a Ponte da Luz em Florianópolis) e ao sul, apresentam maior intensidade de potência gerada (Figura 3a), podendo superar valores de 20 $\mathrm{MW} / \mathrm{dia}$, enquanto que ao norte, é observado valores inferiores a $5 \mathrm{MW} / \mathrm{dia}$, tornando esta região a menos viável. O desvio padrão (Figura 3b) se apresenta alto nos mesmos locais de alta geração de potência, sugerindo que, ao mesmo tempo em que estas regiões são propícias à conversão de energia elétrica, estas também podem passar por períodos de grande variabilidade, inclusive com baixa geração energética, visto que o desvio padrão da velocidade apresenta um valor próximo à média.

A potência máxima observada no ponto I e II chega a $170 \mathrm{~kW} / \mathrm{Dia}$, ou seja, alcança a potência nominal idealizada para a turbina, demonstrando alto potencial para essas regiões. Durante o período de 120 dias analisado, a potência integrada pode alcançar valores em torno de $2.5 \mathrm{MW}$. 


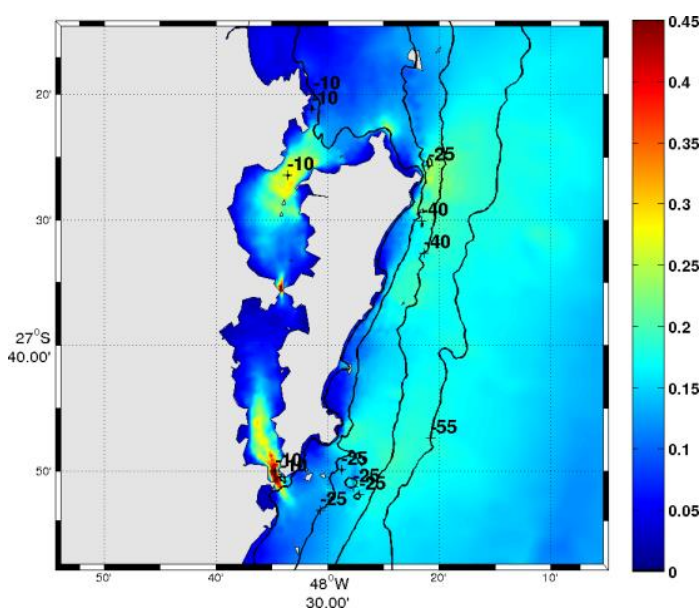

a)

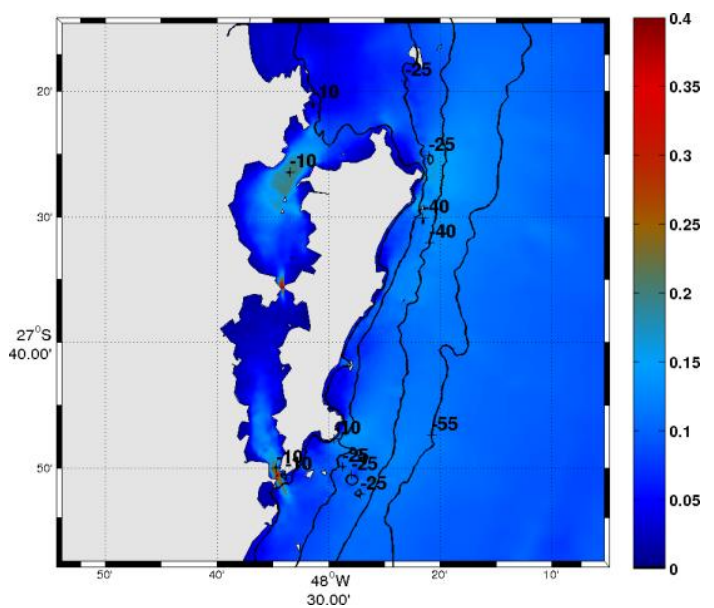

b)

Figura 2: (a) Velocidade média de corrente $(\mathrm{m} / \mathrm{s})$ e seu desvio padrão (b) durante todo o período de simulação.

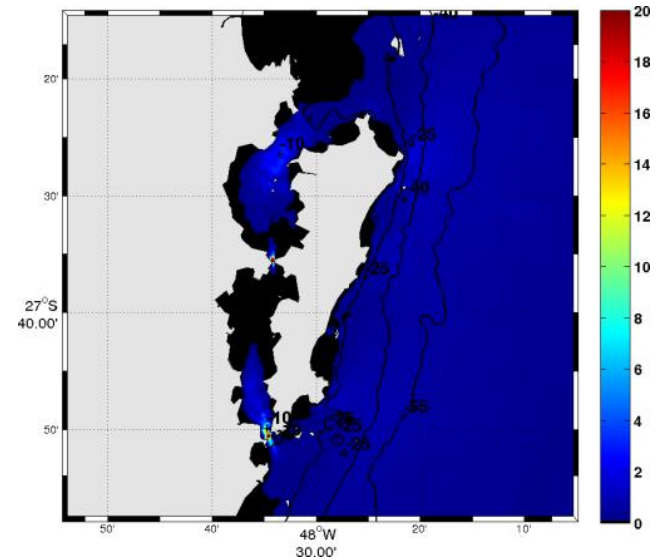

a)

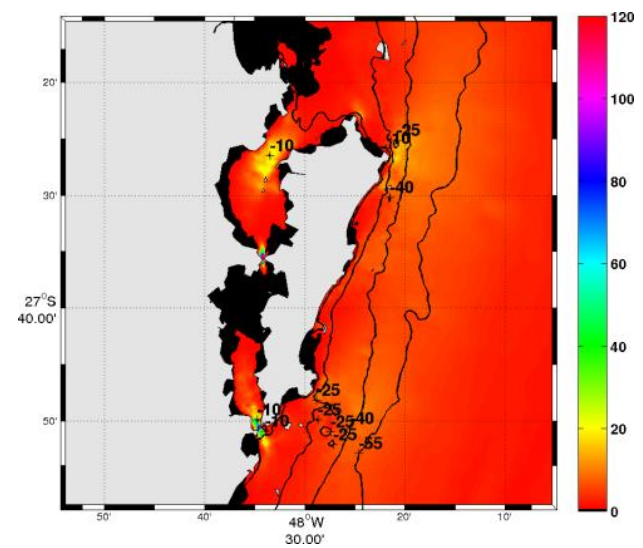

c)

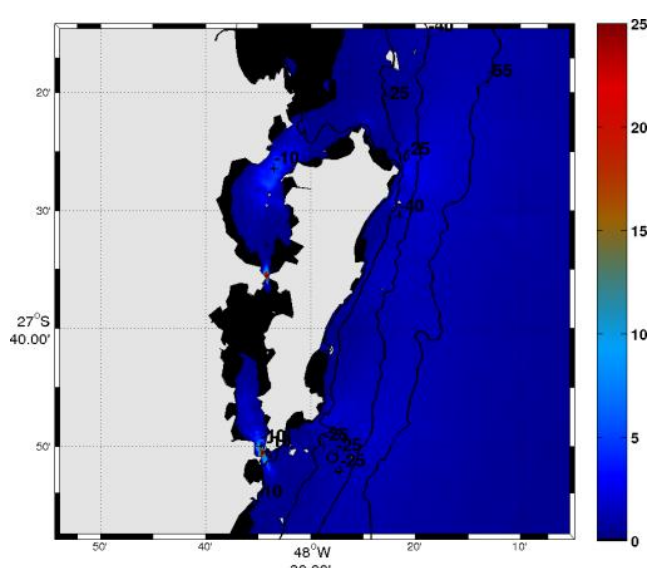

b)

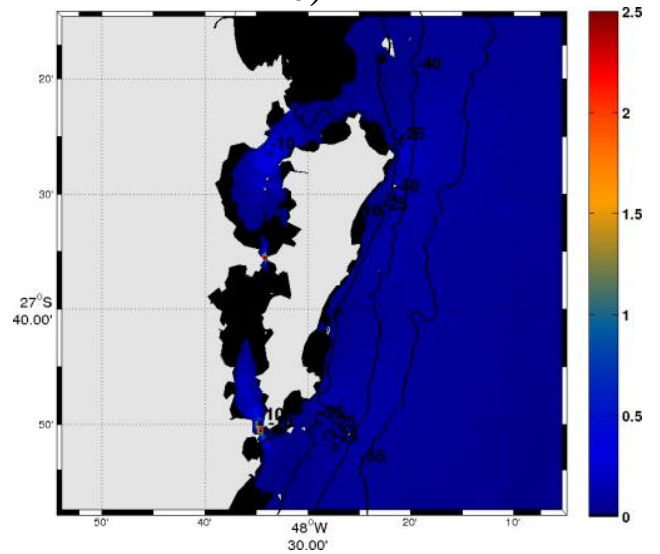

d)

Figura 3: Análise da potência para a região de interesse em Santa Catarina. (a) Energia média (kW/dia); (b) Desvio padrão da potência ( $k W / d i a)$; (c) Energia máxima ( $k W / d i a)$; (d) Energia integrada (MW/120 dias). A linha batimétrica se encontra com valores definidos variando de 0 a $-55 \mathrm{~m}$ de profundidade.

\subsection{Região de Ilhabela - SP}

A região de Ilhabela se destaca no litoral do estado de São Paulo por oferecer uma barreira física (ilha) e um canal que intensifica as correntes nessa região. Sendo assim, na Figura 4, podemos observar valores de velocidade média em torno de $0,3 \mathrm{~m} / \mathrm{s}$ no ponto $\mathrm{V}$, enquanto que no ponto IV, a velocidade gira em torno de $0,25 \mathrm{~m} / \mathrm{s}$. O desvio padrão é observado nas mesmas 
regiões sugerindo uma alta variabilidade da intensidade de corrente. Na Figura 5 serão apresentados os resultados do módulo de conversão de energia para esta região. As regiões em cor preta na figura demonstram baixas ou nenhuma intensidade do parâmetro estudado.

A geração de potência para essa região demonstra valores médios (Figura 5a) em torno de 8 $\mathrm{kW} /$ dia no ponto $\mathrm{V}$, enquanto que no ponto IV, aproximadamente $5 \mathrm{~kW} /$ dia. Nesta região são estimados picos máximos de potência (Figura 5c) alcançando $100 \mathrm{~kW} /$ dia, ou seja, próximo a um terço da potência nominal da turbina idealizada para essa região. Durante os 120 dias de simulação foram estimados a geração de $0,89 \mathrm{MW}$ de potência no ponto $\mathrm{V}$, e na região externa da ilha, 0,61 MW.

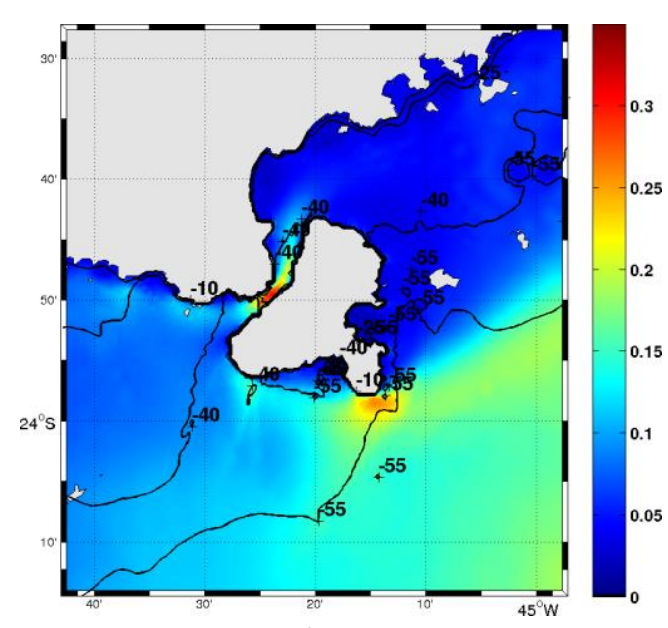

a)

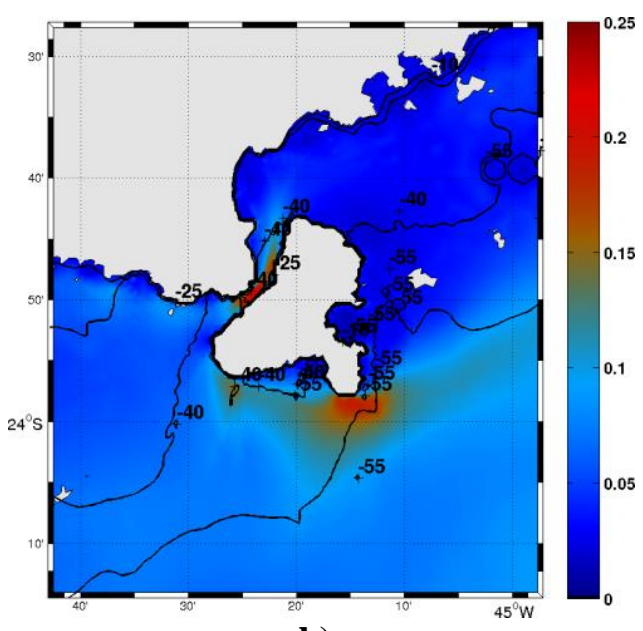

b)

Figura 4: (a) Velocidade média de corrente $(\mathrm{m} / \mathrm{s})$ e seu desvio padrão (b) durante todo o período de simulação.

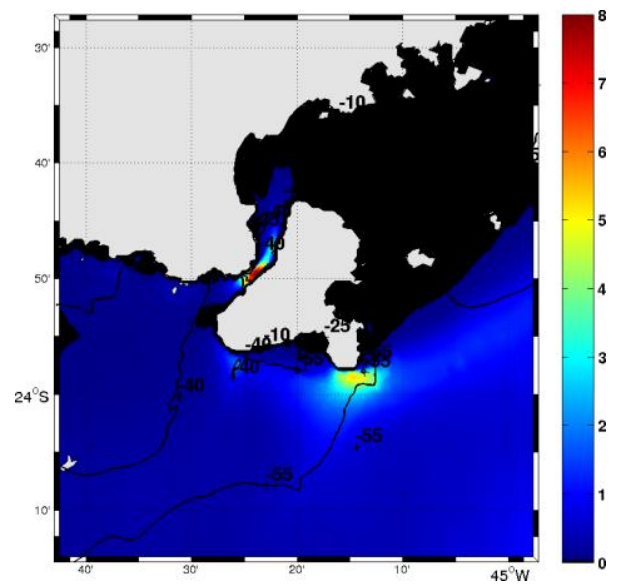

a)

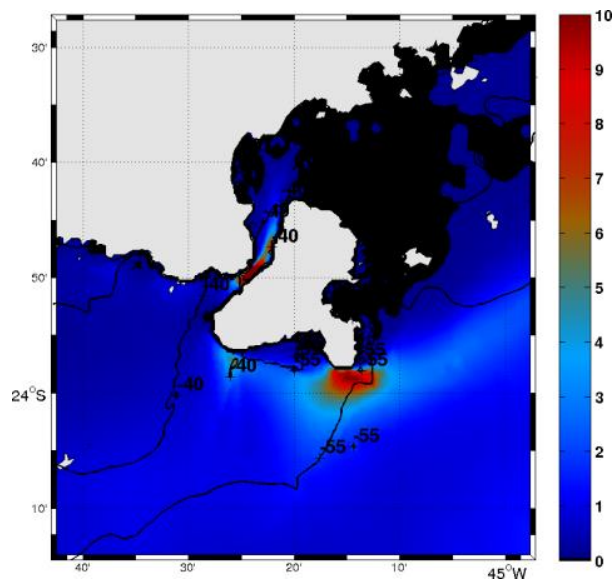

b) 


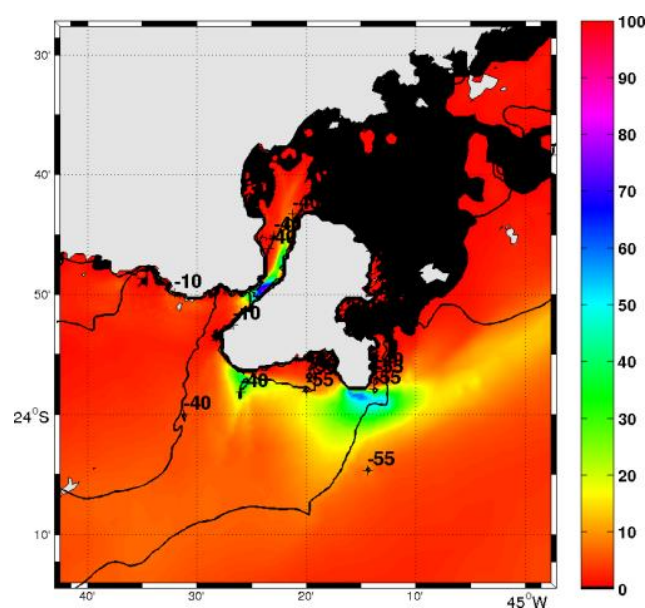

c)

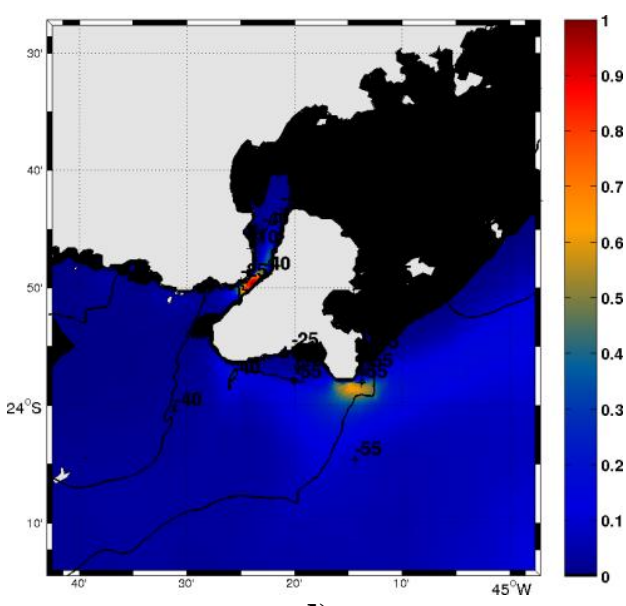

d)

Figura 5: Análise da potência para a região de interesse em São Paulo. (a) Energia média (kW/Dia); (b) Desvio padrão da potência (kW/Dia); (c) Energia máxima (kW/Dia); (d) Energia integrada (MW/120 dias). A linha batimétrica encontra-se com valores definidos variando de 0 a -55m de profundidade

\subsection{Frequência de conversão energética}

As regiões estudadas apresentam diferentes características de velocidade e capacidade de potência gerada, como observado nas sessões anteriores. Entretanto, as taxas de frequência de conversão energética podem auxiliar na identificação dos locais mais energéticos dentro dos parâmetros estabelecidos por este estudo.

Na Tabela 2, é possível observar que a região de Santa Catarina apresenta maiores taxas de produção energética com destaque para o ponto II, alcançando produção superior a $80 \mathrm{~kW} / \mathrm{dia}$ durante $47,50 \%$ do período estudado. Este resultado indica alta estabilidade de conversão provavelmente causada pela constrição no centro da baía de Florianópolis, gerando intensas correntes nesta região. O ponto I também demonstra alto potencial, apresentando em $23 \%$ do período, potência superior a $80 \mathrm{~kW}$. O ponto III apresentou o pior desempenho deste estudo, com produção inferior a $40 \mathrm{~kW}$ durante todo o período estudado.

Para a região de Ilhabela, a frequência de conversão foi baixa, principalmente ocasionada pela intensidade da velocidade inferior a $1 \mathrm{~m} / \mathrm{s}$ durante todo o período estudado. Devido a isso, a potência gerada por essa região ficou subestimada, sendo uma alternativa para estudos futuros realizar alterações na parametrização das turbinas para esta região, buscando intensificar a geração energética.

Tabela 2: Valores de energia e frequência dos diferentes cenários

\begin{tabular}{|c|c|c|c|c|c|}
\hline & $S C$ & & \multicolumn{3}{|c|}{$S P$} \\
\hline Energia & $I$ & II & III & $I V$ & $V$ \\
\hline Média $(k W)$ & 45,55 & 44,05 & 5,46 & 5,09 & 7,48 \\
\hline Desvio padrão $(k W)$ & 84,76 & 64,56 & 10,17 & 10,70 & 13,51 \\
\hline Integrado $(M W)$ & 3,01 & 4,71 & 0,36 & 0,61 & 0,89 \\
\hline Frequência & $I$ & II & III & $I V$ & $V$ \\
\hline$<40 \mathrm{~kW}(\%)$ & 56,66 & 35,00 & 100 & 97,5 & 95,5 \\
\hline $40-80 \mathrm{~kW}(\%)$ & 20,00 & 17,50 & 0 & 2,5 & 5 \\
\hline$>80 \mathrm{~kW}(\%)$ & 23,33 & 47,50 & 0 & 0 & 0 \\
\hline
\end{tabular}




\section{CONCLUSÃO}

Na Plataforma Continental do Sul-Sudeste do Brasil, foram encontradas duas regiões com maior potencial para instalação dos conversores de energia de correntes.

Devido às características das velocidades de correntes e batimetria do local, seria recomenda a utilização de uma turbina helicoidal na região de Santa Catarina, enquanto que na região de Ilhabela, a parametrização da turbina axial idealizada não foi bem sucedida para gerar alto potencial de energia.

A avaliação da viabilidade energética das regiões estudadas demonstrou que o ponto I e II localizados em Santa Catarina apresenta o melhor potencial para a conversão de energia das correntes oceânicas. Valores integrados de potência podem alcançar 4,71 MW durante o período analisado com média de conversão de aproximadamente $45 \mathrm{~kW} /$ dia.

A utilização e captação dessa energia inexplorada no Brasil podem auxiliar a matriz energética brasileira, além de ser uma alternativa energética ambientalmente amigável.

\section{AGRADECIMENTOS}

Os autores do presente trabalho agradecem à Agência Nacional do Petróleo (ANP), ao Programa de Recursos Humanos (PRH-27) e ao Conselho Nacional de Pesquisa (CNPq) pelo financiamento parcial, através do pagamento de bolsas de estudo. Os autores agradecem também ao Conselho Nacional de Pesquisa $(\mathrm{CNPq})$ por dar suporte a este trabalho sob os contratos: 456292/2013-6 e 305885/2013-8.

\section{REFERÊNCIAS BIBLIOGRÁFICAS}

1. Cruz JMBP, Sarmento AJNA. Sea State Characterization of the Test Site of an Offshore Wave Energy Plant. Ocean Eng. 2007;(24):763-75.

2. EPA. State CO2 Emissions from Fossil Fuel Combustion [Internet]. 2005. Available from: http://epa.gov/climatechange/emissions/state_energyco2inv.html

3. Khan MJ, Bhuyan G, Iqbal MT, Quaicoe JE. Hydrokinetic energy conversion systems and assessment of horizontal and vertical axis turbines for river and tidal applications: A technology status review. Appl Energy. Elsevier Ltd; 2009;86(10):1823-35.

4. Gorlov BA. Helical Turbine and Fish Safety. 2010;1-14.

5. Cornett A. Inventory of canada's marine renewable energy resources. Ottawa; 2006 p. 101.

6. Hall DG, Reeves KS, Brizzee J, Sommers GL. Feasibility Assessment of the Water Energy Resources of the United States for New Low Power and Small Hydro Classes of Hydroelectric Plants. 2006.

7. Defne Z. Multi-Criteria assessment of wave and tidal power along the Atlantic coast of the southeastern USA. Georgia Institute of Technology; 2010. p. 209.

8. EPRI. Tidal power in North America Environmental and Permitting Issues. Electric Power Research Institute's . EPRI-TP-007-NA.; 2006.

9. Olson DB, Podestá GP, Evans RH, Brown OB. Temporal variations in the separation of Brazil and Malvinas Currents. Deep Sea Res Part A Oceanogr Res Pap. 1988 Dec.

10. Gordon AL. Brazil-Malvinas Confluence - 1984. Deep Res Part a-Oceanographic Res Pap. 1989;36(3):359-84.

11. Chelton DB, Schlax MG, Witter DL, Richman JG. Geosat altimeter observations of the surface circulation of the Southern Ocean. J Geophys Res. 1990;95(C10):17877.

12. Cirano M, Mata MM, Campos EJ, Deiró NF. A circulação oceânica de larga-escala na região oeste do Atlântico Sul com base no modelo de circulação Global OCCAM. Rev Bras Geofísica. 2006 Jun ;24(2):209-30.

13. Oliveira LR, Piola AR, Mata MM, Soares ID. Brazil Current surface circulation and energetics observed from drifting buoys. J Geophys Res. 2009 Oct 7;114(C10):C10006.

14. Hervouet JM. Free surface flows: Modelling with the finite element methods. England; 2007.

15. Marques WC, Fernandes EHL, Rocha LAO, Malcherek A. Energy converting structures in the Southern Brazilian Shelf: Energy conversion and its influence on the hydrodynamic and morphodynamic processes. Journal of Earth Sciences and Geotechnical Engineering. 2012;1:61-85.

16. Marques WC, Fernandes EHL, Moller OO. 2010a. Straining and advection contributions to the mixing process of the Patos Lagoon coastal plume, Brazil. Journal of Geophysical Research, 115(C6). 
17. Marques WC, Fernandes EHL, Monteiro IO, Möller OO. 2009. Numerical modeling of the Patos Lagoon coastal plume, Brazil. Continental Shelf Research, 29:556-571.

18. Marques WC, Fernandes EH, Möller Jr OO, Moraes BC, Malcherek A. 2010. Dynamics of the Patos Lagoon coastal plume and its contribution to the deposition pattern of the southern Brazilian inner shelf. Journal of Geophysical Research, 115

19. Gorlov BA. Tidal Energy. Acad Press. 2001;2955-60.

20. Douglas CA, Harrison GP, Chick JP. Life cycle assessment of the Seagen marine current turbine. Proceedings of the Institution of Mechanical Engineers, Part M: Journal of Engineering for the Maritime Environment. 2008;222:1-12.

21. Hammons TJ. Tidal Power. Proceedings of the IEEE. 1993. p. 419-33. 